\title{
TARMED-Info
}

Bulletin $n^{\circ} 4$

Rédaction TARMED*

- Structure tarifaire: la procédure d'approbation du Conseil fédéral des conventions tarifaires TARMED porte à confusion.

- Négociations tarifaires: examen des contrats cantonaux d'affiliation à la convention cadre TARMED par des juristes et des médecins du Groupe G7

- Tarif LAA: cf. «Structure tarifaire».

- Interface: concepts de physiothérapie et tarification des prestations physiothérapeutiques en cabinet médical et dans le domaine ambulatoire.

\footnotetext{
* Markus Baumgartner,

Hans Heinrich Brunner,

Andreas Häfeli,

Annamaria Müller Imboden,

Denise Rüegg,

Reto Steiner;

Coordination: Markus Trutmann.
}

1 Cf. TARMED-Info $n^{\circ} 3$. Bull Méd Suisses 2002;83(27):1435-6.

Correspondance:

Bulletin des médecins suisses

Rédaction TARMED

Case posatale

CH-4010 Basel

e-mail: tarmed@emh.ch

\section{Structure tarifaire}

Le Conseil fédéral a renoncé à approuver les conventions tarifaires TARMED avant les vacances d'été et a, du même coup, bloqué l'approbation de la version 1.1 du TARMED. D'après les informations internes dont nous disposons, cette décision aurait été motivée par le fait que la convention-cadre signée et juridiquement valable entre la FMH et santésuisse n'aurait été présentée au Conseil fédéral qu'à la mi-juin et de manière incomplète, autrement dit sans la convention concernant la Commission paritaire d'interprétation TARMED (CPI) ${ }^{1}$.

Une telle argumentation a de quoi étonner, en effet:

- La convention AA/AM/AI a été soumise au Conseil fédéral au début de cette année déjà et n'attend plus que la signature des offices fédéraux soumis au DFI, à savoir l'Office fédéral de l'assurance-militaire et l'Office fédéral des assurances sociales.

- La version 1.1 de la structure tarifaire TARMED a été soumise au Conseil fédéral pour approbation le 22 avril 2002, simultanément aux concepts concernant la valeur intrinsèque, les unités fonctionnelles et les projets de convention concernant la nouvelle organisation TARMED («TARMED SUISSE»). Bien que, dans l'intervalle, la convention concernant TARMED Suisse ait été signée par toutes les parties concernées (donc également par les deux offices fédéraux précités!), la structure organisationnelle prévue ne pourra pas être mise en œuvre, étant donné que «l'objet à mettre en œuvre», la structure tarifaire, n'a pas encore été approuvé. Les parties concernées, y compris la FMH, n'ont donc provisoirement pas d'autre choix que de continuer à travailler au sein des anciennes structures de projet peu efficaces, ce qui ne saurait faciliter les travaux de remaniement (RE II).

- La convention critiquée concernant la CPI doit encore être signée non seulement par les parties à la convention-cadre LAMal, mais également par tous les partenaires TARMED, étant donné qu'elle fait partie des tâches de la nouvelle organisation TARMED Suisse et qu'elle doit être signée par toutes les parties à la convention, donc également par les offices fédéraux de l'assurance militaire et des assurances sociales. Certains ne veulent cependant signer la convention concernant la CPI que lorsque le Conseil fédéral aura approuvé les conventions AA/AM/AI. En d'autres termes, c'est le serpent qui se mord la queue.

En résumé, force est de constater que la procédure d'approbation du Conseil fédéral porte à confusion et peut faire croire que les différentes étapes d'approbation s'entravent mutuellement.

\section{Négociations tarifaires}

Les contrats cantonaux d'affiliation à la convention cadre TARMED sont actuellement examinés par des juristes et des médecins du Groupe G7. Ils seront adressés avec les commentaires, à fin juillet 2002 pour la version allemande et au début août 2002 pour la version française, à tous les présidents des sociétés cantonales de médecine. Ceux-ci auront l'occasion de se prononcer à leur sujet jusqu'à fin août 2002.

\section{Tarif LAA}

Cf. «Structure tarifaire». 


\section{Interface}

Concepts de physiothérapie et tarification des prestations physiothérapeutiques en cabinet médical et dans le domaine ambulatoire

Dans ce contexte, vous voudrez bien vous référer au bulletin $n^{\circ} 2$ de TARMED-Info. Bull Méd Suisses 2002;83(23):1179-81.

\section{Coopération de la FMH aux concepts FISIO}

Après une première séance en avril 2002, les sociétés et sociétés de discipline ont été invitées à continuer à coopérer activement au développement des deux concepts FISIO «concept de qualité» et «indication à la physiothérapie».

Jusqu'ici, les sociétés suivantes ont annoncé leur coopération: Société suisse de médecine manuelle (SAMM), Société suisse de médecine générale (SSMG), Société suisse de médecine interne (SSMI), Société suisse d'orthopédie (SSO), Société suisse de pédiatrie (SSP), Société suisse de médecine physique et réadaptation (SSMPR), Société suisse de rhumatologie (SSR), Société suisse de médecine du sport (SSMS) et Union des sociétés suisses de maladies vasculaires.

Les premières prises de position, à savoir celles de la SSR, de la SSMPR et de la SSMG, ont été communiquées à toutes les sociétés intéressées pour information. La priorité a été donnée aux questions de l'indication à la physiothérapie et à la communication respectivement la coopération au cabinet médical entre médecin et physiothérapeute.

Les sociétés intéressées ont été invitées à prendre position, d'ici la mi-octobre 2002, sur les concepts FISIO. Le Dr Beat Dejung, membre du Comité de la SSR et représentant de cette société et de la SSMPR, prendra en mains la coordination des diverses prises de position. Toutes les sociétés affiliées ont été de plus conviées à participer à la prochaine séance avec les cadres de l'Association suisse des physiothérapeutes (ASP). Cette séance aura lieu le 7 novembre 2002 à Berne. La FMH devrait donner son aval, fin 2004, aux deux concepts FISIO «concept de qualité» et «indication à la physiothérapie».

Si vous souhaitez la participation d'autres sociétés ou sociétés de discipline à l'élaboration des concepts FISIO, vous voudrez bien nous le communiquer (e-mail: pbonfils@hin.ch).

\section{Tarification des prestations FISIO au cabinet médical}

Simultanément à l'élaboration des concepts, il s'agit de traiter la question de la tarification des prestations de physiothérapie au cabinet médical.
Les médecins de cabinets médicaux dans lesquels travaille du personnel qualifié en physiothérapie, peuvent facturer en principe toutes les prestations relevant du tarif «FISIO». Par contre, les cabinets médicaux sans personnel qualifié ne peuvent facturer que les positions pouvant être exécutées par du personnel non qualifié.

Depuis le 8 mai 2002, les présidents des sociétés et sociétés de discipline médicale concernés au premier chef ont été invités directement et plusieurs fois à nous communiquer le plus vite possible les prestations de physiothérapie qu'ils revendiquent pour leur discipline ou, plus précisément, quelles prestations physiothérapeutiques ambulatoires doivent pouvoir être facturées dans les cabinets médicaux sans personnel qualifié dans cette discipline. Le Collège suisse de médecine de premier recours (CMPR), la Foederatio Medicorum Curantium (FMC) et la Foederatio Medicorum Scrutantium (FMS) ont été informés en permanence.

Le Bulletin $\mathrm{n}^{\circ} 2$ de TARMED-Info a donné la liste des sociétés directement contactées, donnant en même temps aux autres sociétés la possibilité de participer. Quelques sociétés parmi lesquelles la Société suisse d'orthopédie, la Société suisse de neurologie et la Société suisse de cardiologie, n'ont pas envoyé de prise de position.

Le tarif des prestations FISIO contient 7 positions pour les forfaits de séances, 6 positions supplémentaires et 6 positions concernant le maintien des droits acquis.

Les sociétés suivantes ont envoyé des propositions concrètes: la Société suisse de médecine générale (SSMG), la Société suisse de chirurgie de la main (SSCM), la Société suisse de médecine interne (SSMI), la Société suisse de médecine manuelle (SAMM), la Société suisse de pédiatrie (SSP), la Société suisse de médecine physique et réadaptation (SSMPR), la Société suisse de pneumologie (SSP), la Société suisse de rhumatologie (SSR) et l'Union des sociétés suisses de maladies vasculaires.

Plusieurs positions tarifaires du tarif FISIO ont été demandées par les sociétés susmentionnées. Certaines de ces demandes sont liés à une valeur intrinsèque, une attestation de formation complémentaire ou une formation postgraduée et continue. La question du maintien des droits acquis a également été abordée.

Les prestations FISIO dites complexes ne figurent néanmoins pas dans le tarif FISIO. Il appartenait aux sociétés intéressées de définir ces prestations éventuelles, le cas échéant de quantifier leurs propositions. Des propositions concrètes ont été faites de la part de la Société suisse de 
pédiatrie (SSP), de la Société suisse de médecine physique et réadaptation (SSMPR), de la Société suisse de rhumatologie (SSR) et de l'Union des sociétés suisses de maladies vasculaires.

En coopération avec le Dr Andi Wüest, membre du Comité de la SSR et représentant de la SSR et de la SSMPR, nous sommes en train d'examiner toutes les propositions et de prendre les mesures qui s'imposent.

Selon l'interprétation générale 49 du TARMED 1.1: «Sont applicables, pour les prestations de physiothérapie, les conventions tarifaires bilatérales passées entre partenaires». Dans ce contexte, il s'agit d'adapter le projet antérieur - valable pour l'ensemble de la Suisse - de convention tarifaire FISIO pour le domaine AA établi entre la FMH et la Commission des tarifs médicaux pour l'AA (CTM), l'assurance-invalidité (AI), représentée par l'Office fédéral des assurances sociales (OFAS) et l'Office fédéral de l'assurance militaire (OFAM). De nombreuses questions demeurent ouvertes et doivent trouver leur solution.

Physiothérapie dans le domaine ambulatoire Le 11 juillet 2000, un groupe de projet a remis au support organisationnel TARMED un concept intitulé TarReha, destiné à la tarification multiprofessionnelle et non-médicale de prestations de réadaptation dans le domaine ambulatoire.

A l'initiative de H+ (les hôpitaux de Suisse), ce concept pourrait éventuellement être repris. Dans ce cas, la FMH s'est d'ores et déjà déclarée disposée à coopérer activement, aussi bien à l'échelle du comité d'intervention que dans le cadre de l'organisation du projet. Après examen et adaptation éventuelle, notamment de la situation initiale, des objectifs, du mandat et des conditions-cadre du concept jusqu'ici en vigueur, il s'agira entre autres de différencier, dans le secteur ambulatoire, les questions de réadaptation des questions de physiothérapie.

\section{FAQ}

L'introduction du TARMED est-elle forcément liée à un passage du tiers garant au tiers payant? Seul le système du tiers payant permet une facturation efficace par voie électronique.

Non. Le système du tiers garant permet également la facturation par voie électronique. Le changement éventuel de système devra être décidé dans les conventions cantonales entre sociétés de médecine et association des caisses-maladie.

Les interprétations générales évoquent le fait que la facturation électronique ne peut se faire qu'avec le système standard XML. Est-ce vraiment raisonnable de fixer des standards dans une structure tarifaire?

Non. Le standard XML n'a jamais été discuté dans la forme entre partenaires des négociations. Les standards de facturation électronique doivent être définis au sein des conventions. La délégation de la FMH s'efforce de corriger cette erreur avant l'introduction du tarif. 